\title{
Health Impacts of Floods
}

\author{
Paul George, MA (Disaster Management)
}

Tata Institute of Social Sciences, Mumbai, India

Prehosp Disaster Med 2011;26(2):137

Correspondence:

E-mail: paulgeorgeps@gmail.com

Online publication: 3 May 2011

doi: 10.1017/S1049023X11000148
The article "Health Impacts of Floods" published in Volume 25, Number 3 of Prehospital and Disaster Medicine is a commendable effort in identifying the health impacts during floods and their causes. I would like to draw special attention to the section about the variance in the health impacts on communities. The authors have done well in mentioning the patterns of exposure as a crucial component to the determination of the health impacts.

In more developed nations with better response systems and health infrastructure, the role of the patterns of exposure in determining health effects are relatively less significant than in the developing world. Better sanitation and hygiene would negate a large chunk of the communicable diseases. Monitoring and warning systems alert the populations to flash floods and facilitate evacuation. Developed communication and transportation would make evacuation, response, and relief much easier. However, in nations with poor public health and emergency response systems in place, the past exposure of a community to floods is a very big determinant. This is a context in which the state is unable to provide those basic services, so it is entirely up to the community to develop means to cope with contingencies. This development of coping mechanisms that limit the impacts of the hazard can occur naturally only through repeated exposure to the hazard.

Repeated exposure to floods leads to the adoption of coping mechanisms and resilience to floods for the community. Such frequently exposed communities cope much better with the impacts of floods, including those related to health. The Mhising community of Assam and the floods in Bihar in 2008 illustrate this point. The former is in an area that is flooded by the Brahmaputra for at least five months of the year. There is only a low number of fatalities, and post-flood communicable diseases such as diarrheal diseases are combated by the promotion of hygiene and safe sanitation practices. Over time, this tribal community has developed many structural innovations and other nonstructural mechanisms to mitigate the risks of floods without much external assistance or intervention. To say that floods are an integral part of life in this community would not be an exaggeration.

However, in 2008 the state of Bihar experienced floods in five districts of a magnitude not witnessed in decades. In the absence of a set warning and response system, the floods took a large toll on the affected areas. Many were washed away, and lives were lost due to the spread of communicable diseases and lack of medical facilities in the relief camps. The official death toll was 239 , and a large number of livestock was washed away.

Therefore, in countries with struggling infrastructure, while designing and conducting health interventions amongst flood affected communities it is essential to take into account the patterns of exposure as a determinant in limiting or exacerbating the impacts of the flood. 